\title{
ANTICIPATION OF DETERIORATING HEALTH AND INFORMATION AVOIDANCE
}

Johannes Schünemann

Holger Strulik

Timo Trimborn

Georg-August-Universität Göttingen 


\title{
Anticipation of Deteriorating Health and Information Avoidance
}

\author{
Johannes Schünemann ${ }^{\dagger}$, Holger Strulik ${ }^{\ddagger}$ and Timo Trimborn ${ }^{\S}$
}

February 2019.

\begin{abstract}
The anticipation of bad future events reduces currently experienced happiness and it may through this channel elicit detrimental behavioral responses. We explore this idea in the context of endogenous health and aging. We integrate physiological aging into a life-cycle model, calibrate it with data from gerontology, and analyze how the anticipation of a deteriorating state of health affects health spending, life expectancy, and the value of life. In counterfactual computational experiments we compare behavior and outcomes of anticipating and non-anticipating individuals and find that anticipation decreases lifetime utility, health investments, and longevity. We then use the model to contribute to the literature on information avoidance. We find that anticipation provides a strong motive to avoid medical testing even when the likelihood of developing a certain disease is high and the cost for the test is low.

Keywords: Health, Anticipation, Longevity, Health Behavior, Value of Life, Information Avoidance
\end{abstract}

JEL: D11, D91, I12, J17

\footnotetext{
* We would like to thank Friedrich Breyer, Alexia Fürnkranz-Prskawetz, Volker Grossmann, Michael Kuhn, and Peter Zweifel for discussion and helpful comments.

$\dagger$ University of Goettingen, Department of Economics, Platz der Goettinger Sieben 3, 37073 Goettingen, Germany; email:

¥ University of Goettingen, Department of Economics, Platz der Goettinger Sieben 3, 37073 Goettingen, Germany; email: holger.strulik@wiwi.uni-goettingen.de.

§ Aarhus University, Department of Economics and Business Economics, Fuglesangs Allé 4, 8210 Aarhus V, Denmark; email: tt@econ.au.dk..
} 


\section{INTRODUCTION}

It is a well-established fact that health is an important component in the evaluation of people's well-being. The health status of an individual varies over the life course and, in particular, declines in the course of human aging. From a health perspective, the human life cycle can be described as the continuous deterioration of physiological fitness, which eventually culminates in death (Arking, 2006; Case and Deaton, 2005; Skirbekk, 2004; Nair, 2005). It seems natural that, controlling for other factors in the utility function, people become less happy when new health deficits arrive. In this paper, we shall argue that it is not only the actual appearance of health deficits that influences health behavior and health outcomes, but also the anticipation of (not yet existing) future health deficits affecting instantaneous utility already today.

The psychology of anticipatory feelings has been acknowledged and analyzed in various settings. Lazarus (1966) surveys the experimental literature showing that certain forms of physical pain, such as pin pricks, do not cause any distress beyond the mere anticipation of those events. By means of survey techniques, Loewenstein (1987) finds that the willingness to pay for avoiding an electric shock delayed by one up to ten days was substantially higher than for avoiding an immediate shock. These studies imply that people experience much higher disutility from anticipating the shock than from the negative shock itself. Most importantly for our study, the detrimental effect of anticipation has also been established in the medical literature. A natural way of identifying anticipatory effects in the context of future health deficits is to look at the effect of diagnoses on measures of happiness before symptoms of the disease have set in. Honiden et al. (2006), for example, find a negative impact of HIV diagnosis on health-related utility. Likewise, Cuypers et al. (2017) report a detrimental effect of prostate cancer diagnosis on health-related quality of life.

The first goal of the paper is to determine the impact of health deficit anticipation on health behavior, longevity, and happiness. The cleanest way of identifying this effect would be to compare health behavior and outcomes of an anticipating individual to that of an otherwise identical non-anticipating individual in the same environment. Obviously, this strategy is impossible to carry out by means of lab or field experiments due to the missing counterfactual. With the help of a theory-based computational experiment, however, an assessment is relatively straightforward. In this paper we follow such an approach and set up a life-cycle model of human aging. We calibrate the model with gerontological data and compare behavior and outcomes 
of anticipating and non-anticipating individuals. We find that anticipating types spend less on health and live shorter than otherwise identical non-anticipating types. We use these results and compute the implied lifetime utility or welfare of the individuals. According to our benchmark calibration, anticipation of aging reduces lifetime utility by around $11 \%$.

At this point we should explain how we distinguish anticipation from expectation and how we extend the available health economic theory. In any dynamic economic model, individuals form expectations about the future and these expectations affect their current behavior. Conventional economic models, however, assume that the expectation of future events has no impact on currently experienced (i.e. instantaneous) utility. Anticipation, in contrast, means that the expectation of bad future events affects instantaneous utility already today. Through this channel, it may then elicit further behavioral changes that may amplify or dampen those evoked by the mere "cold" expectation of these events. We apply this notion of anticipation in a dynamic model of health and aging where individuals are assumed to experience a decline in instantaneous utility today from health deficits developed in the future.

We use our model of health deficit anticipation to contribute to the discussion on information avoidance. Interestingly, people tend to avoid medical testing even if the likelihood of developing a certain condition is high and the cost of the test is low. Shoulson and Young (2011) and Oster et al (2013a) report testing rates of 5-10 \% among individuals at high risk for Huntington's disease. Similar, although less dramatic patterns arise in the case for cancer screening (Ropka et al., 2006; Lerman et al. 1999), genetic testing for Alzheimer's disease (Roberts et al., 2004) and HIV testing (Thornton, 2008). Standard health economic models cannot explain this observation. In these models, having more information on future health shocks is always beneficial since individuals are able to adjust and re-optimize their behavior. We argue that including anticipation of future health deficits into the utility function induces people to avoid being tested. Although individuals can re-optimize their decisions after being diagnosed, they experience an instantaneous utility loss from knowing that they will become ill. This loss is quantitatively substantial. We estimate the willingness to pay for avoiding diagnosis of Huntington's disease at the age of 20 to be around $\$ 260,000$ or nine annual wages.

The model that we develop below in order to discuss the effects of anticipation of deteriorating health is particularly suitable for this purpose since it conceptualizes aging as the progressive accumulation of health deficits. The alternative paradigm, the Grossman (1972) model, is less 
suitable since it is based on health capital accumulation. Besides structural shortcomings, health capital is a latent variable, unknown to doctors and medical scientists, which confounds any serious calibration of the model. The health deficit model, based on Dalgaard and Strulik (2014), in contrast, is founded in gerontological research which enables us to calibrate it straightforwardly using the so-called frailty index (Mitnitski et al., 2002a,b). Other studies employing the health deficit model investigate the role of adaptation for health behavior (Schünemann et al., 2017a), the gender gap in mortality (Schünemann et al., 2017b), optimal aging in partnerships (Schünemann et al., 2017c), the historical evolution of retirement (Dalgaard and Strulik, 2017), and the education gradient (Strulik, 2018).

There exists a strand of theoretical literature dealing with anticipatory effects in the utility function. Loewenstein (1987) and Monteiro and Turnovsky (2016) introduce utility from anticipated consumption while Koszegi (2003, 2006), and Oster et al. (2013a), among others, analyze anticipation in a health context. To the best of our knowledge, however, we are the first to analyze the impact of anticipation in a life-cycle framework with endogenous health and longevity in which anticipation is modeled as a stock variable dependent on the actual evolution of future health. This allows us to study the anticipatory effects on life-cycle health behavior, longevity, the value of life, and information avoidance in a qualitatively and quantitatively convincing way.

The paper is organized as follows. Section 2 presents the basic model of health deficit anticipation for two different types of individuals, which we will call anticipating and non-anticipating types. In Section 3, we calibrate the model to health behavior and outcomes of a 20 years old reference U.S. American in the year 2010. In Section 3 we analyze how anticipation affects health investments, longevity, and welfare and analyze comparative dynamics with respect to the model parameters. In Section 4, we contribute to the discussion on information avoidance and apply our model to provide a quantitatively meaningful explanation for why people refuse to get tested. Section 5 concludes.

\section{The BAsic Model}

Consider an individual who derives utility from consumption and from being in good health. The (objective) state of health is measured by the accumulated health deficits $D$. Following Dalgaard and Strulik (2014), we assume that deficits accumulate in the course of aging according 


$$
\dot{D}=\mu\left(D-A h^{\gamma}-a\right)
$$

where $\mu$ represents the "natural" rate of aging. The accumulation of deficits can be slowed down by deliberate (monetary) health investments $h$. The scale parameters $A$ and the curvature parameter $\gamma$ govern the health technology with $A>0$ and $0<\gamma<1$, while a captures environmental influences beyond individual control. The individual dies when it has accumulated a critical deficit level $D_{T}$, thereby implying that death is deterministic. In related work, we show that accounting for a more complicated stochastic setup has only little effect on the results (Strulik, 2015; Schünemann et al., 2017).

Apart from the actual health state, utility is also affected by the anticipation of future health deficits. We model the "stock" of anticipation, denoted by $R$, as a weighted average of future health deficits according to

$$
R=D_{T} e^{-\theta(T-t)}+\theta \int_{t}^{T} e^{-\theta(\tau-t)} D(\tau) \mathrm{d} \tau
$$

where $T$ is the time of death and $\theta$ captures the discounting of future health deficits. Therefore, a higher $\theta$ implies that deficits accumulated farther in the future receive a lower weight in the anticipation stock. The first term in equation (2) accounts for the anticipation of death, which will become more pronounced the closer the individual approaches the terminal health deficit level $D_{T}$. Note also that at the time of death $T$, the final level of the anticipation stock coincides with the final deficit level and thus $R(T)=D_{T}$. Our conceptualization of deficit anticipation is related to the modeling of consumption anticipation in Monteiro and Turnovsky (2016), which turns out to be also technically convenient. Differentiating equation (2) with respect to time provides the following simple differential equation:

$$
\dot{R}=\theta(R-D)
$$

Following empirical evidence by Finkelstein et al. (2013), we assume that bad health affects both utility and marginal utility of consumption. Specifically, the instantaneous utility of the 
individual is given by

$$
U(c, D, R)=\left(\frac{\bar{D}}{D}\right)^{\alpha}\left(\frac{\bar{D}}{R}\right)^{\beta} \cdot \tilde{u}(c), \quad \text { with } \tilde{u}(c)=\left\{\begin{array}{ll}
\frac{c^{1-\sigma}-1}{1-\sigma} & \text { for } \sigma \neq 1 \\
\log (c) & \text { for } \sigma=1
\end{array} .\right.
$$

The actual health state as well as the anticipated future health state is evaluated relative to the state of best health $\bar{D}$. The parameter $\alpha$ captures by how much an additional health deficit affects utility, while $\beta$ governs the impact of a unit increase in the anticipation stock.

The individual receives labor income $w$ which can be spent on consumption, health services and savings. We suppose that the individual has access to financial markets and can save or borrow at net interest rate $r$. The budget constraint thus reads

$$
\dot{k}=w+r k-c-p h,
$$

where $p$ denotes the relative price of health investments. The individual chooses $c$ and $h$ to maximize lifetime utility

$$
V=\int_{0}^{T} u(c, D, R) e^{-\rho t} \mathrm{~d} t
$$

subject to (1), (3)-(5), as well as the initial conditions $D(0)=D_{0}$ and $k(0)=k_{0}$, and the terminal conditions $D(T)=D_{T}, k(T)=k_{T}$, and $R(T)=R_{T}=D_{T}$. The parameter $\rho$ represents the time preference rate of the individual. The time of death $T$ is endogenous. Through their health expenditure plan, individuals influence the accumulation of health deficits and thus the time of death, which occurs when $D_{T}$ deficits have been accumulated.

The Hamiltonian associated with this life-cycle problem is given by

$$
\mathcal{H}=u(c, D, R)+\lambda_{k} \dot{k}+\lambda_{D} \dot{D}
$$

where $\lambda_{k}$ and $\lambda_{D}$ denote the shadow prices of capital and deficits. ${ }^{1}$ In order to identify the impact of anticipation on health behavior, we compare behavior of anticipating types to that of nonanticipating individuals. The two types can be distinguished by setting $\theta>0$ for anticipating

\footnotetext{
${ }^{1}$ The evolution of the anticipation stock does not appear in the Hamiltonian. In analogy to Schünemann et al. (2017a)'s study of adaptation to poor health, this behavior can be characterized as naive anticipation. It means that individuals do not deliberately control their anticipation but take the evolution of $R$ as given. In contrast, in the case of sophisticated anticipation, the differential equation for anticipation would appear in the Hamiltonian. In such a scenario, individuals understand that through their behavior they can deliberately affect the anticipation stock. Schünemann et al. (2017a) concluded that naive adaptation better describes actual health behavior.
} 
types, and $\theta=0, R=\bar{D} \forall t$ for non-anticipating types. In the latter case, the anticipation stock equals initial health deficits over the whole life cycle so that utility is not affected by anticipation. The transversality condition for this free-terminal-time problem is given by $H(T)=0$.

The following dynamic equations for consumption and health investment (8) and (9) are derived in the Appendix. From the first-order conditions for the maximization of $\mathcal{H}$, we obtain the Euler equation for consumption growth

$$
\frac{\dot{c}}{c}=\frac{r-\rho-\alpha \frac{\dot{D}}{D}-\beta \frac{\dot{R}}{R}}{\sigma} .
$$

In case $\alpha=\beta=0$, consumption growth is equal to that in the standard life-cycle model. In case $\alpha>0$, health matters for the individual in the utility function and deficit accumulation slows down consumption growth. The reason behind this result can be found in the healthconsumption complementarity. Since a deteriorating state of health reduces the marginal utility of consumption, individuals substitute future for present consumption in order to consume when the marginal utility of consumption is still high. Since deficit anticipation enters utility qualitatively in the same way as actual health deficits, anticipation affects consumption growth symmetrically.

The Euler equation for health expenditure growth is obtained as

$$
\frac{\dot{h}}{h}=\frac{r-\mu+\frac{\alpha U}{D \lambda_{D}}}{1-\gamma}
$$

If $\alpha=0$ and health does not affect utility, equation (9) equals the standard Euler equation for health investments developed in Dalgaard and Strulik (2014). In this case, health investments increase over the life cycle if the interest rate exceeds the rate of aging. If $\alpha>0$, health expenditure growth declines as people substitute future for present health investments to enjoy a good state of health already at the beginning of their life. To see this, note that health deficits are a "bad" rather than a "good", implying that the shadow price $\lambda_{D}$ and thus the last term in the numerator in equation (9) is negative.

2.1. Model Calibration. We solve the model numerically using a shooting procedure ${ }^{2}$. For this purpose, we calibrate the model to a 20 years old male U.S. American in the year 2010. As far as the biological parameters are concerned, the regression analysis by Mitnitski et al. (2002a) employing the frailty index provides us with most of the parameter values. The frailty

\footnotetext{
${ }^{2}$ Details on the solution procedure are provided in the Appendix.
} 
index is suggested by gerontologists as a straightforward metric to measure the state of health. It includes various health deficits ranging from mild nuisances (e.g. reduced vision) to fatal disorders (e.g. cancer). The frailty index is then constructed as the proportion of deficits an individual has from a set of potential deficits. Naturally, the frailty index increases as a function of age. In order to quantify the state of best health $\bar{D}$, we set it equal to the initial state of health when the individual is born in our model, i.e. $\bar{D}=D(0)$. We back out $D(0)=0.0274$ as the relevant initial deficit level associated with a man of age 20 which is the starting age in our model. The terminal state is given by $D(T)=0.1059$ which is associated with a man 57.1 years later; the life expectancy at age 20 for males was 57.1 years in 2010 (i.e. death at 77.1 years; NVSR, 2017). Since $R(T)=D(T)$, the same value applies for the final level of the anticipation stock. From the same study, we take $\mu=0.043$ as the value for the natural rate of aging. Moreover, we take the estimates for the environmental constant $a=0.013$ and the curvature parameter of the health technology $\gamma=0.19$ directly from Dalgaard and Strulik (2014). We further set $r=0.07$ according to the long-run interest rate from Jorda et al. (2017), normalize the relative price of health services to one $(p=1)$ and set $w=27,928$ according to data on wages and salaries for U.S. American single men in 2010 (BLS, 2012).

We simultaneously estimate the remaining six parameters $\rho, \sigma, \alpha, \beta, A$, and $\theta$ by fitting the following six data points: (i) a stable consumption path over the life cycle (see e.g. Browning and Ejrnæs, 2009), (ii) the average life expectancy of 20 years old men, (iii) health expenditures at age 30, 50, and 70 (MEPS, 2010), and (iv) a reduction of health-related utility of $8 \%$ following a HIV diagnosis (Honiden et al., 2006). The study of Honiden et al. (2006) measures how healthrelated utility is affected by a HIV diagnosis, before symptoms and treatment have set in. The survey is carried out for HIV diagnoses in the 1980s and 1990s, a time where there was basically no effective treatment for HIV. Without treatment, the median time from seroconversion to AIDS has been found to be 9 years, while death occurs one year later (Nakagawa et al., 2013). In order to determine the anticipation parameter, we therefore model a health shock which is diagnosed at the age of 35 (the mean age at diagnosis in Honiden et al.'s study), sets in 9 years later and leads to death after another year. We then adjust parameters such that the shock implies a loss in health related utility, captured in our model by $\left(\frac{\bar{D}}{D}\right)^{\alpha}\left(\frac{\bar{D}}{R}\right)^{\beta}$, of $8 \%$ at the time of diagnosis. The calibration results and the externally set biological parameters are shown in Table 1. 
Table 1a: Calibration Results

\begin{tabular}{llllll}
\hline \hline$\rho$ & $\sigma$ & $\alpha$ & $\beta$ & $A$ & $\theta$ \\
\hline 0.065 & 1.16 & 0.01 & 0.22 & 0.00146 & 0.10 \\
\hline \hline
\end{tabular}

Table 1b: Externally Set Parameters

\begin{tabular}{lllll}
\hline \hline$D_{0}$ & $D_{T}$ & $\mu$ & $a$ & $\gamma$ \\
\hline 0.0274 & 0.0159 & 0.043 & 0.013 & 0.19 \\
\hline \hline
\end{tabular}

While some of these parameters are latent and thus cannot be compared to empirical estimates in the literature, our estimate for $\sigma$ fits well the findings in previous studies that the "true" value of $\sigma$ is probably close to unity (e.g. Chetty et al., 2006) or slightly above unity, around 1.2 (Layard et al., 2008). Our calibrated value for $\alpha$ implies that a one-standard-deviation increase in deficits is associated with a reduction in the marginal utility of consumption of $0.4 \%{ }^{3}$ Finkelstein et al. (2013) report a point estimate of this effect of $11 \%$, with a confidence interval ranging from $2.7 \%^{-}$ 16.8\%. Therefore, our parameter value is below the lower bound of Finkelstein et al.'s confidence interval. When estimating the effect of health on the marginal utility of consumption, however, Finkelstein et al. do not take into account the effect of anticipation which makes our results not directly comparable to those obtained in their study. Another reason for the discrepancy between the estimates may lie in the fact that the Finkelstein et al. study only uses a narrow set of very severe health deficits while the frailty index includes a wide range of health deficits of varying severity. We consider the effects of higher $\alpha$ 's in a comparative dynamics analysis in Section 2.2 .

Comparing the parameter values of $\alpha$ and $\beta$ reveals that the individual experiences much more disutility from anticipating future deficits than from developing actual health deficits. This observation is consistent with the statement made in the introduction that the anticipation of electric shocks and pin pricks is more harmful for the individual than the negative shock itself. Calibrating a theory of health anticipation to actual data, we confirm this notion also in the context of human aging and the associated accumulation of health deficits.

\footnotetext{
${ }^{3}$ Given the average life expectancy applied in our calibration of 77.1 years, the frailty index in Mitnitski et al. (2002) implies a deficit mean of 0.0521 and a standard deviation of 0.0221. Starting from the mean, a one-standard-deviation increase in deficits reduces the marginal utility of consumption by $0.04 \%$ for $\alpha=0.01$.
} 
As far as the anticipation parameter $\theta$ is concerned, Monteiro and Turnovsky (2016) who model anticipation of consumption in a structurally equivalent way to ours draw on habit formation studies and set $\theta=0.2$ in their numerical analysis. Besides the fact that consumption anticipation cannot be directly compared to the anticipation of future health deficits, in the habit formation literature the reference stock is a weighted average of past consumption levels, while in an anticipation framework we consider a future stock of consumption streams. To the best of our knowledge, we are the first to calibrate the anticipation parameter $\theta$ to actual data.

2.2. Results. In this section we look at the impact of anticipation on health behavior and outcomes in the course of aging, i.e. for the gradual increase of deficits when people get older. This allows us to flesh out the basic mechanisms and the economic intuition behind the effects of anticipation. ${ }^{4}$ Figure 1 shows the results for the age trajectories of the anticipation stock, health investments, health deficits, utility, and consumption and capital for two individuals. Life cycle trajectories for the calibrated anticipating benchmark American are represented by blue (solid) lines and blue dots denote data points. Red (dashed) lines represent the counterfactual: a non-anticipating individual, as commonly modeled in economic life cycle theory, who faces otherwise the same parameters as the benchmark American.

The upper-left panel of Figure 1 shows the evolution of the anticipation stock. While for the non-anticipating type the anticipation stock is, by construction, constant and equal to $\bar{D}$, the anticipation stock of the anticipating individual increases in the course of aging. Inspecting equation (2), there are two mechanisms how the stock of anticipation is affected as people age. First, the lower bound of the integral increases, which, taken for itself, reduces the anticipation stock since the time span at which future deficits are accounted for decreases. Second, the number of health deficits increases with age, implying that in the course of aging health states with a high number of deficits come closer and are discounted at lower rates. This increases the anticipation stock and it is the dominating effect as shown in Figure 1.

As can be seen from the upper-right panel in Figure 1, health expenditure increases over the life cycle as observed empirically (MEPS, 2010). The panel also shows that the model manages to fit actual expenditure data reasonably well. Interestingly, anticipating types spend less on their health at any age. As a consequence, anticipating individuals accumulate health deficits at

\footnotetext{
${ }^{4}$ In this section, we focus on anticipation of aging as gradual accumulation of health deficits. We do not yet analyze any abrupt health shocks. The HIV shock considered in the calibration section only served for pinning down the anticipation parameters. In the benchmark run carried out in this section, there will be no such shock.
} 
Figure 1: Health Anticipation: Benchmark Run
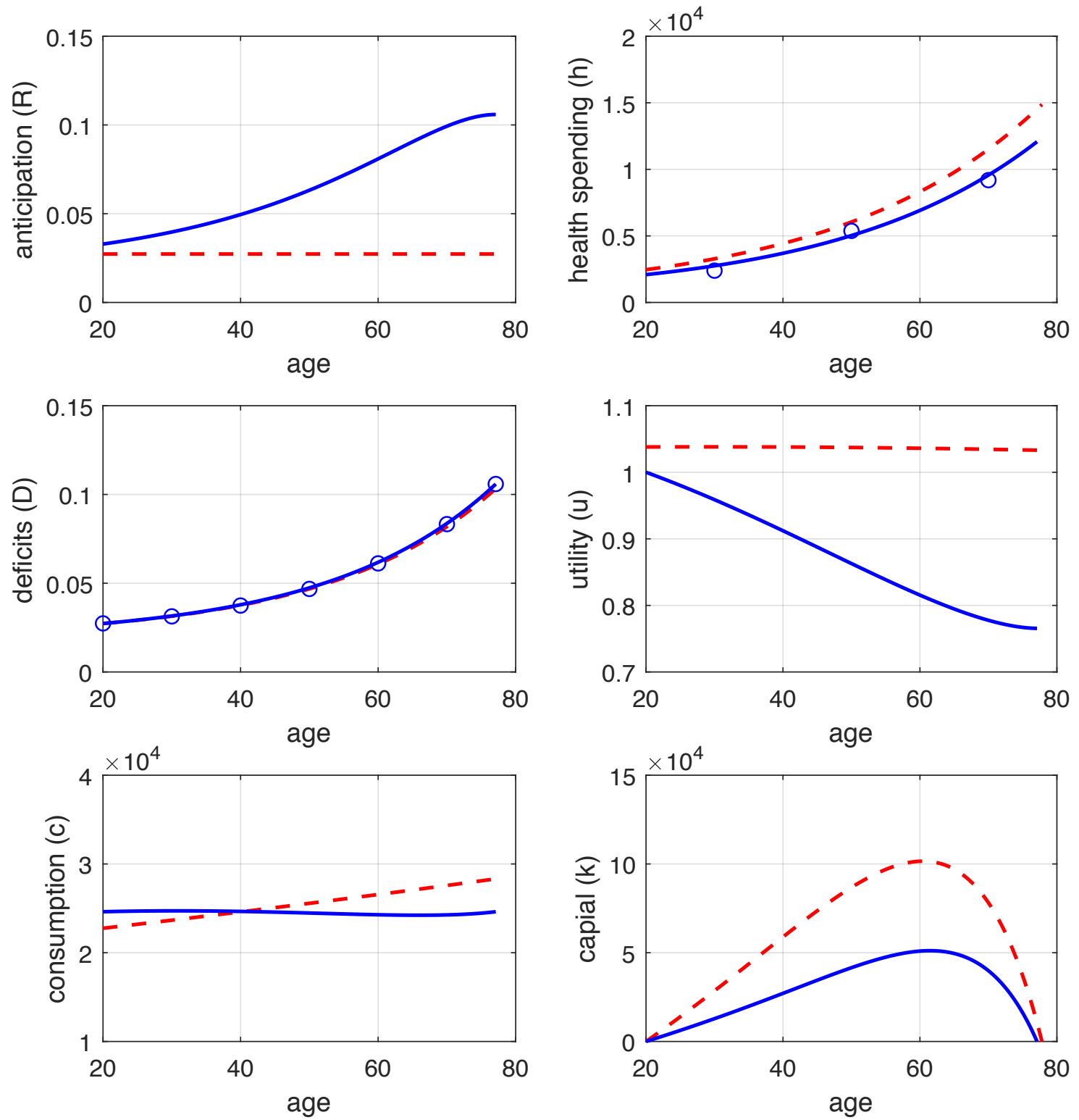

Blue (solid) lines: anticipating type. Red (dashed) lines: non-anticipating type. Utility is instantaneous utility relative to initial utility of an anticipating individual. Dots indicate data points. Data for health spending are from MEPS (2010) and data on deficits are from the frailty index in Mitnitski et al. (2002).

a higher pace as shown in the third panel and thus die earlier. Table 2 shows the effect of anticipation by calculating the percentage deviation of the counterfactual non-anticipating individual from the benchmark anticipating type with respect to discounted lifetime health expenditure, life expectancy at age 20, and welfare. Welfare refers to lifetime utility of the individual as defined in equation (6). As can be seen from the first row of Table 2, discounted lifetime health 
expenditure of non-anticipating types is almost $20 \%$ percent higher than that of anticipating types. Consequently, non-anticipating individuals have a $1.26 \%$ higher life expectancy at age 20 than anticipating individuals. The anticipation of future health events makes anticipating types more unhappy as it reduces instantaneous utility. Since life is less worthwhile at any age, anticipating types invest less in prolonging life compared to non-anticipating types. This mechanism is illustrated in the fourth panel of Figure 1. The panel shows instantaneous utility in the course of aging. Since anticipating types draw negative utility from anticipating future health deficits, their utility declines more rapidly over life compared to non-anticipating types. Life-time utility is found to be almost $11 \%$ higher for non-anticipating individuals.

Table 2: Main Results

\begin{tabular}{llrrr}
\hline \hline case & remark & health exp. & life expect. & welfare \\
\hline benchmark & & 19.95 & 1.26 & 10.62 \\
$\beta=0.1$ & lower utility weight of anticipation & 8.68 & 0.59 & 4.76 \\
$\beta=0.3$ & higher utility weight of anticipation & 28.78 & 1.72 & 14.98 \\
$\theta=0.07$ & lower discounting of anticipated health & 17.35 & 1.10 & 13.25 \\
$\theta=0.13$ & higher discounting of anticipated health & 21.43 & 1.33 & 9.18 \\
$\alpha=0.1$ & higher utility weight of health & 14.56 & 1.08 & 9.97 \\
$\alpha=0.2$ & higher utility weight of health & 10.87 & 0.96 & 9.44 \\
$\Delta w=-50 \%$ & lower income & 20.32 & 0.96 & 11.50 \\
$\Delta w=+50 \%$ & higher income & 19.71 & 1.48 & 9.99 \\
\hline \hline
\end{tabular}

All values as deviation in percent from the optimal solution for an anticipating individual; health exp. is the discounted lifetime expenditure on health; life expect. is life expectancy at 20; welfare is lifetime-utility as in (6).

The fifth panel of Figure 1 illustrates the age trajectories for consumption. According to our calibration strategy, the consumption path for the anticipation type is fairly stable while consumption increases over the life cycle for non-anticipating types. The consumption Euler equation (8) provides the explanation for this observation. As shown above, the growth rate of the anticipation stock enters consumption growth in a negative way and (additionally) counterbalances a positive consumption growth trend (from $r>\rho$ ). This effect is missing for nonanticipating individuals, implying positive consumption growth compared to the anticipating individual. The last panel shows how the capital stock evolves over time for the two individuals. Non-anticipating individuals tend to accumulate more wealth than anticipating types. Since non-anticipating types spend more on health especially in old age and exhibit an increasing 
consumption path compared to anticipating individuals, they have to save more at young ages to finance their expenditure plan in the future.

In the next four rows of Table 2 we analyze some comparative statics of the model with respect to the anticipation parameters. To this end, we check how value changes of $\beta$ and $\theta$ affect health behavior and outcomes. Inspecting the cases of $\beta=0.3$ and $\beta=0.1$, we see that the effect of anticipation increases in the size of $\beta$. A higher value of $\beta$ raises the weight of anticipation in the utility function and amplifies the negative impact of anticipation on health expenditure, life expectancy, and welfare compared to the non-anticipating type and vice versa. A higher value of $\theta$ reduces the welfare gain from no anticipation, i.e. the welfare loss from anticipation declines. The reason behind this result is that future health deficits are discounted at a higher rate in the buildup of the anticipation stock, which is thus lower at any age. The opposite result holds true for a reduction in $\theta$.

Rows five and six consider the effect of higher values of $\alpha$ and thus a higher weight of health in the utility function. We successively increase $\alpha$ to 0.1 and 0.2 implying that a one-standarddeviation increase in deficits is associated with a $3.5 \%$ and $6.8 \%$ reduction in the marginal utility of consumption, respectively. A higher $\alpha$ reduces the impact of anticipation on health expenditure and longevity since with a higher weight of health in utility, the weight of anticipation in utility becomes relatively smaller and thus anticipation has a smaller impact on health behavior. Despite the large variation in the parameter value, however, varying $\alpha$ alters the impact of anticipation on welfare only marginally. The last two rows show comparative dynamic results with respect to income $(w)$. Changing the wage rate by $50 \%$ demonstrates that anticipatory effects are only mildly affected by income. Naturally, higher income increases longevity and instantaneous utility of both types, but in relative terms, the effect of anticipation is only moderately affected by different levels of income.

\section{Expected vs. Unexpected Health Shocks and Information Avoidance}

In this section, we use our model of health anticipation to provide an explanation for information avoidance in the context of health. Our starting point is the seminal study by Oster et al. (2013a) on genetic testing among individuals at risk for Huntington's disease (HD). HD is a neurological disorder that is genetically inherited. Individuals with one parent carrying the expansion in the Huntington gene will eventually develop the disease with 50\% probability. 
Although the cost for genetic testing is fairly small, the authors find considerably low testing rates (below 10\%) among individuals at risk, i.e. among individuals with a family background of HD. Similar findings are reported for the case of cancer screening (Ropka et al., 2006; Lerman et al. 1999), genetic testing of Alzheimer's disease (Roberts et al., 2004), HIV testing (Thornton, 2008), and again for HD (Shoulson and Young, 2011). This pattern is hard to reconcile with standard economic theory where more information is always beneficial since individuals can adjust and re-optimize their behavior. We argue that the disutility from anticipating future health events induces people to refuse to learn about future health shocks.

The idea that anticipation may lead to information avoidance is not an entirely new one. Several previous studies have acknowledged that the combination of low testing rates and low testing costs may be motivated by future beliefs affecting utility directly (e.g. Koszegi, 2003; Caplin and Leahy, 2001, 2004). Oster et al. (2013a) propose an optimal expectation model based on Brunnermeier and Parker (2005) with anticipatory utility in order to explain the low testing rates found in their data. However, these models do not investigate anticipation in a life-cycle model of endogenous health and longevity in which the actual health state has a microfoundation in gerontology. Including anticipation as a state variable dependent on the actual evolution of future health allows us to quantify the detrimental effect that the diagnosis of a future disease has on welfare and behavior of the individual.

To this end, we model the case of HD and compare lifetime utility after an expected health shock, i.e. when being diagnosed with HD at the age of 20, to an unexpected health shock when the individual is not being tested before the disease sets in. At this point we should take up the discussion of the introduction again and elaborate on the distinction between anticipation and expectation. A future health shock is said to be expected if the individual knows before that the shock will eventually set in, while a health shock is considered unexpected if the individual is hit by the shock without previous knowledge. An individual is said to be anticipating if an expected future health shock reduces instantaneous utility already today, while an individual is considered non-anticipating if a future health shock has no impact on instantaneous utility today. Consequently, an unexpected future health shock neither affects today's instantaneous utility of the anticipating nor the non-anticipating type because people are not aware that the health shock will eventually arrive. The implementation of unexpected health shocks will be our means to model an individual which carries HD and does not get diagnosed. One might think 
that this experiment does not perfectly mimic the case of a HD carrier not being tested because the individual may know that it will develop the disease with $50 \%$ probability, which in turn may influence future behavior. However, Oster et al. (2013) find that untested individuals at risk behave similarly to those who are certain not to carry the genetic expansion. With expected health shocks we will model HD carriers who get diagnosed before and thus know that they will develop HD at some point in the future. In this case, anticipating individuals experience a reduction in instantaneous utility at the time of the diagnosis (and before the disease sets in), while instantaneous utility of non-anticipating types remains unaffected by the diagnosis. However, a positive diagnosis may still change health behavior of the non-anticipating type in order to prepare for the upcoming shock.

According to Oster et al. (2013a), the onset of HD is around the age of 40 while death occurs on average 20 years later at the age of 60 . Therefore, we model a health shock at 40 such that the age at death of the anticipating type declines from 77.1 years to 60 years. In terms of our model, this amounts to an increase in the deficit level at 40 of $0.7 \cdot D_{0}$, i.e. $70 \%$ of the initial deficit level. Figure 2 shows the results for this experiment with respect to the anticipation stock, instantaneous utility, and health expenditure. Blue (solid) lines represent the benchmark run, red (dashed) lines show the model response to the expected health shock, and green (dashdotted) lines the results for the unexpected health shock. ${ }^{5}$ The upper panels of Figure 2 show the non-anticipating individual and the lower panels show the anticipating individual. Starting with the non-anticipating individual, we see that, by construction, the anticipation stock is constant at $R=D_{0}$. Since future health deficits do not enter instantaneous utility directly, the results for instantaneous utility look quite similar for the expected and unexpected health shock (the difference is around 0.1-0.2\%). At age 40, in both cases utility declines on impact when HD sets in. The age trajectories for health expenditure, however, depend on whether the individual knows that it will become ill or not. If the disease is diagnosed at the age of 20 , the individual immediately responds by increasing health investments at any age in order to counteract the upcoming health shock. If the individual does not know that it carries the genetic expansion, it behaves as in the benchmark case until age 40. When the disease sets in, non-diagnosed individuals increase health investments on impact above the level of the expected health shock scenario to compensate for the time in which they did not adjust their behavior.

${ }^{5}$ Details on the numerical procedure are provided in the Appendix. 
Figure 2: Expected vs. Unexpected Health Shocks
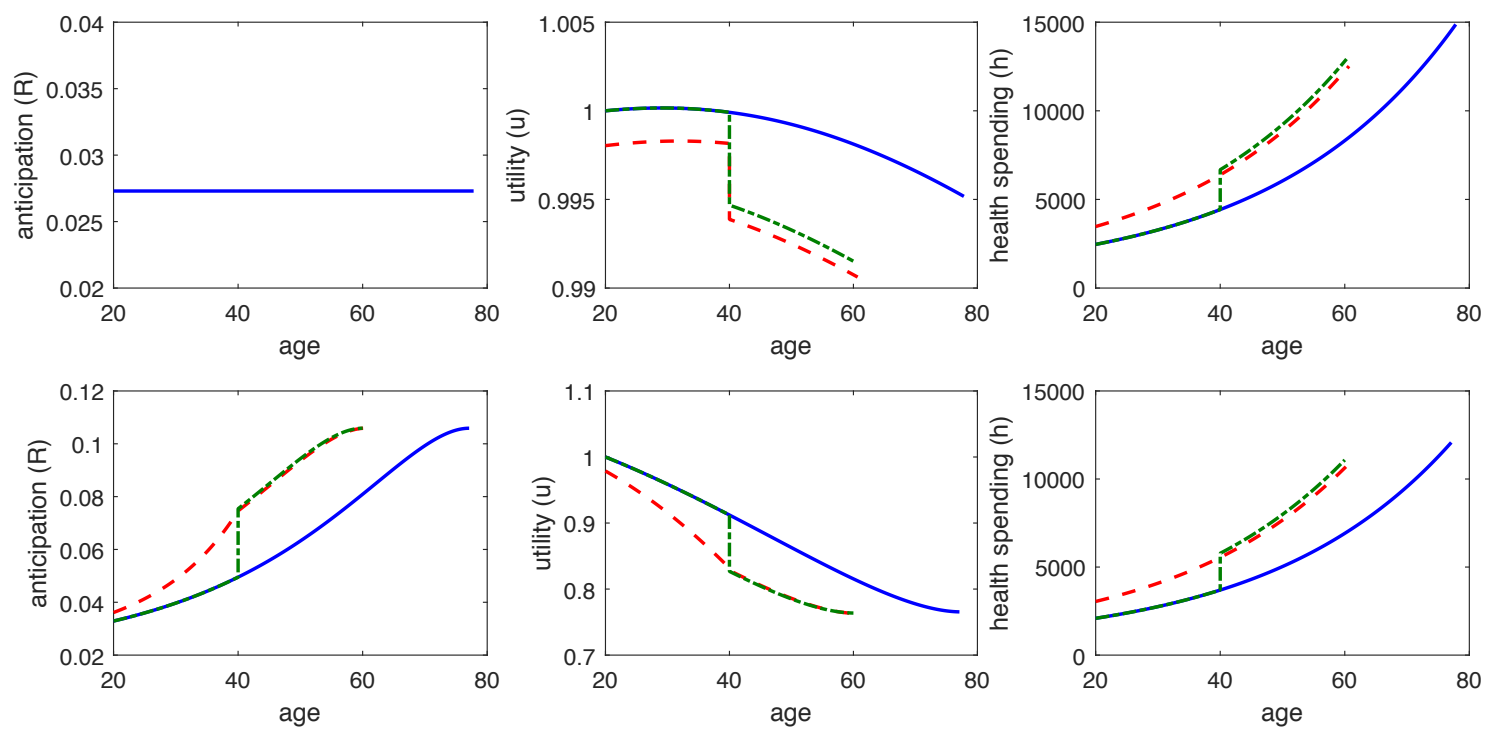

Blue (solid) lines: benchmark. Red (dashed) lines: expected shock. Green (dash-dotted) lines: unexpected shock. The upper panels show results for the non-anticipating type and the lower panels for the anticipating type. Utility is instantaneous utility relative to initial utility of the benchmark run.

Turning to the anticipating individual, the blue (solid) line in the first lower panel reiterates the benchmark run for the anticipation stock of the anticipating type which we already analyzed in Figure 1. Looking at the unexpected health shock, the anticipation stock coincides with the benchmark run until the age of 40 and then increases on impact when HD sets in. If the individual is diagnosed with HD at the age of 20 , however, the anticipation stock increases gradually already from the beginning as the knowledge of the future evolution of HD-related health deficits enters the anticipation variable. Since anticipation affects utility, this also translates into the age trajectories for instantaneous utility illustrated in the second lower panel. Again, for the unexpected shock utility decreases on impact when the disease sets in at the age of 40 . If the individual learns about the HD diagnosis at age 20, utility declines already right from the beginning due to the disutility caused by anticipating the future health shock. At age 40, utility additionally jumps down on impact following the sudden increase in actual health deficits. The last panel shows the effects of the the health shock on health investments which are qualitatively similar to those of the non-anticipating type.

Table 3 shows the quantitative results for the two outcome variables life expectancy and lifetime utility or welfare. The first pair of columns includes the shocks for the non-anticipating type while the second pair looks at the anticipating individual. The numbers are provided 
as percentage deviation from the benchmark run without any health shock. In all cases, the reduction in life expectancy amounts to around 30\%, which in absolute terms implies a decrease of 17.1 years as calibrated above (the difference between average longevity of 77.1 years and average longevity of an individual with HD of 60 years). Interestingly, in case of the nonanticipating type, there is only a small difference between the expected and unexpected shock in terms of welfare. Although in the unexpected shock scenario the individual can only start reoptimizing at the age of 40, he experiences virtually the same lifetime utility as when the shock is known at the age of 20 . This picture changes, however, when we look at the anticipating type. Being diagnosed at the age of 20 results in a 3 percentage-point lower lifetime utility than being hit by the disease surprisingly. Consequently, the individual would choose to avoid being tested and rather live an additional 20 years without the burden of knowing that HD will eventually set in.

Table 3: HD at age 40: Impact on Life Expectancy and Welfare

\begin{tabular}{lcccc}
\hline \hline & \multicolumn{2}{c}{ no anticipation } & \multicolumn{2}{c}{ anticipation } \\
outcome & expected & unexpected & expected & unexpected \\
\hline 1) LE & -29.64 & -30.34 & -29.19 & -29.95 \\
2) Welfare & -5.10 & -5.13 & -8.89 & -5.93 \\
\hline \hline
\end{tabular}

All values as deviation in percent from the benchmark run without health shock; expected denotes the expected health shock and unexpected the unexpected health shock.

With our model at hand, we can also determine the willingness to pay in order to avoid positive testing or, in other words, the monetary compensation value for receiving a HDpositive diagnosis. For this purpose, we calculate the difference in the Value of Life (VoL) between experiencing the expected and the unexpected shock. The VoL converts lifetime utility measured in "utils" into monetary equivalents and is given by the expression $V O L=$ $\left.\int_{0}^{T} \mathrm{e}^{-\rho \tau} u[c(\tau), D(\tau), R(\tau)] \mathrm{d} \tau\right) / u_{c}[c(0), D(0), R(0)]$ where $u_{c}$ denotes the marginal utility of consumption. The VoL of our reference anticipating individual is 8.73 million dollars. This value is in line with empirical estimates which find the VoL to range from 7 million (Murphy and Topel, 2006, Fig. 3) to 10 million dollars (Moran and Monje, 2016).

Results are reported in Table 4. The welfare difference between the expected and unexpected shock from Table 3 is now expressed in monetary values. As the table shows, the individual needs a compensation of 258,532 dollars in order to be indifferent between knowing and not knowing that HD will eventually set in. In other words, the individual would forgo around nine 
Table 4: $\Delta$ Value of Life (in $\$$ )

\begin{tabular}{cc}
\hline \hline age at diag. & $V o L_{\text {unex }}-V o L_{e x}$ \\
\hline 20 years & 258,532 \\
30 years & 134,615 \\
\hline \hline VoL $L_{\text {unex }}-V o L_{e x}$ & refers to the welfare \\
difference between the expected and the \\
unexpected shock expressed in dollars.
\end{tabular}

annual wages to lead an unburdened live until age 40 . In column 2, we report results from the same experiment when the individual learns the diagnosis at age 30 instead of 20. Naturally, the willingness to pay for avoiding a positive diagnosis decreases in the time that the individual has to spend with anticipating the future health shock reduces.

\section{CONCLUSion}

In this paper we set up a gerontologically founded life-cycle model of human aging in which the anticipation of future health deficits enters instantaneous utility. We calibrated the model to a 20 years old U.S. American and compared anticipating individuals to otherwise identical non-anticipating individuals with respect to health expenditure, life expectancy, and welfare. We found that anticipation reduces investments in health and thus life expectancy since the detrimental effect of anticipating future health deficits makes life less worthwhile. Accordingly, we showed that lifetime utility is lower for anticipating individuals.

We then applied our model of health deficit anticipation to the literature on information avoidance in the context of health. We illustrated that anticipatory feelings are a quantitatively powerful explanation for why people refuse to be tested even if testing costs are low. For our benchmark case, we estimated that individuals at the age of 20 would forgo around nine annual wages to avoid a Huntington's disease diagnosis.

Our framework could be applied to analyze other life-cycle decisions. A natural model extension would be to investigate unhealthy consumption patterns in the light of anticipation. As Oster et al. (2013b) point out, being diagnosed with Huntington's disease reduces the probability to quit smoking. Moreover, anticipation of future health deficits may also play a role for the retirement decision since anticipating individuals are expected to have a shorter working life. 


\section{Appendix A: Derivation of the Euler Equations}

The first-order conditions associated with the optimal control problem read:

$$
\begin{aligned}
\frac{\partial \mathcal{H}}{\partial c} & =0 \Leftrightarrow \lambda_{k}=\left(\frac{D_{0}}{D}\right)^{\alpha}\left(\frac{D_{0}}{R}\right)^{\beta} c^{-\sigma} \\
\frac{\partial \mathcal{H}}{\partial h} & =0 \Leftrightarrow p \lambda_{k}=-\lambda_{D} \mu A \gamma h^{\gamma-1} \\
\frac{\partial \mathcal{H}}{\partial D} & =-\dot{\lambda}_{D}+\lambda_{D} \rho \\
& \Leftrightarrow \frac{\dot{\lambda}_{D}}{\lambda_{D}}=\rho-\mu+\frac{\alpha}{D \lambda_{D}} u(c, D, R) \\
\frac{\partial \mathcal{H}}{\partial k} & =-\dot{\lambda}_{k}+\lambda_{k} \rho \\
& \Leftrightarrow \frac{\dot{\lambda}_{k}}{\lambda_{k}}=\rho-r .
\end{aligned}
$$

Log-differentiating (A.1) w.r.t. time and using (A.4) provides:

$$
\begin{aligned}
\frac{\dot{\lambda}_{k}}{\lambda_{k}} & =-\alpha \frac{\dot{D}}{D}-\beta \frac{\dot{R}}{R}-\sigma \frac{\dot{c}}{c} \\
\Leftrightarrow \rho-r & =-\alpha \frac{\dot{D}}{D}-\beta \frac{\dot{R}}{R}-\sigma \frac{\dot{c}}{c} .
\end{aligned}
$$

Solving (A.5) for consumption growth provides equation (8) in the main text.

Log-differentiating (A.2) w.r.t. time and using (A.3) and (A.4) we obtain:

$$
\begin{gathered}
\frac{\dot{\lambda}_{k}}{\lambda_{k}}=\frac{\dot{\lambda}_{D}}{\lambda_{D}}+(\gamma-1) \frac{\dot{h}}{h} \\
\Leftrightarrow \rho-r=\rho-\mu+\frac{\alpha}{D \lambda_{D}} u(c, D, R)+(\gamma-1) \frac{\dot{h}}{h} .
\end{gathered}
$$

Using (A.1) and (A.2) and solving (A.6) for health expenditure growth provides equation (9) in the main text.

\section{Appendix B: Solution Method}

We start by deriving the following dynamic system

$$
\begin{aligned}
\dot{D} & =\mu\left(D-A h^{\gamma}-a\right) \\
\dot{R} & =\theta(R-D) \\
\dot{k} & =w+r k-c-p h
\end{aligned}
$$




$$
\begin{aligned}
& \dot{\lambda}_{D}=-\lambda_{D}(\rho-\mu)+\frac{\alpha}{D} u(c, D, R) \\
& \dot{\lambda}_{k}=\lambda_{k}(\rho-r)
\end{aligned}
$$

To solve for the optimal life-cycle trajectories we apply a shooting algorithm. This type of algorithm is frequently used to solve differential equations for which only some of the initial conditions are given and additionally a set of final boundary conditions has to be satisfied, i.e. the problem is a two-point boundary value problem. The general idea of shooting is to guess the unknown initial values of the variables and calculate a trial solution by integrating the dynamic system for a given time span. Then, the initial values are updated in an iteration process until the final boundary conditions are met as well. We have to adapt the standard shooting to our setting since the length of the time span is endogenous and determined by a transversality condition.

Noticing that the initial values for the state variables $D$ and $k$ are given, we have three degrees of freedom for choosing initial conditions. We provide initial guesses for $R, \lambda_{D}$, and $\lambda_{k}$. We then solve system (10) with the standard Matlab routine for initial value problems (ode45.m) until the individual dies at $D_{T}$. There are, however, three conditions that the trial solution does not satisfy: The transversality condition $\mathcal{H}(T)=0$ and the final boundary conditions $R(T)=D_{T}$ and $k(T)=0$. We then adjust the initial conditions until these three additional conditions are met by using a Newton-Raphson algorithm.

\section{Appendix C: Expected vs. Unexpected Shocks}

In Section 3, we contrast health shocks that the individual expects with health shocks that set in unexpectedly. Numerically, we proceed in the following way. In order to model the unexpected health shock at age 40, we separately solve for the pre- and post-shock period and merge the two solutions in the end. For the first part, we solve for the optimal solution of the benchmark scenario with no shock. We truncate the solution at the age of 40 and use the terminal values of $k$ and $D$ as initial values for the second part. For the second part, we then add to the initial value of $D$ the amount of deficits that is associated with Huntington's disease $\left(0.7 * D_{0}\right.$ deficits in our case) and let the individual solve for the optimal trajectories again. In the end, we merge the two parts at age 40 . 
Regarding the expected shock, there exist necessary conditions which have to hold at the time of the shock:

$$
\lambda_{k}\left(t_{0}-\right)=\lambda_{k}\left(t_{0}+\right), \quad \lambda_{D}\left(t_{0}-\right)=\lambda_{D}\left(t_{0}+\right)
$$

where $t_{0}-$ denotes the time just before the shock and $t_{0}+$ the time just after the shock. In other words, the shadow prices for capital and deficits are continuous at the time of the shock. We include these interior boundary conditions into our numerical solution procedure and solve for the optimal trajectories when the same shock as above occurs at the age of 40 . One direct implication of the continuity of the shadow prices is that health investments behave smoothly at the age of the shock, while after the unexpected shock health investments jump on impact (see Figure 2). 


\section{REFERENCES}

Alvarez-Cuadrado, F., Monteiro, G., and Turnovsky, S. J. (2004). Habit formation, catching up with the Joneses, and non-scale growth. Journal of Economic Growth 9(1), 47-80.

Arking, R. (2006). The Biology of Aging: Observations and Principles, Oxford University Press, Oxford.

BLS (2012). Consumer Expenditure Survey. U.S. Bureau of Labor Statistics, September 2012 (http://www.bls.gov/cex/csxcross.htm\#y1011).

Browning, M., and Ejrns, M. (2009). Consumption and children. The Review of Economics and Statistics 91(1), 93-111.

Brunnermeier, Markus, K., and Jonathan A. Parker. 2005. Optimal expectations. American Economic Review, 95 (4): 1092-1118.

Caplin, Andrew, and Leahy, John (2001). Psychological expected utility theory and anticipatory feelings. Quarterly Journal of Economics 116(1), 5579.

Caplin, Andrew, and Leahy, John (2001). The supply of information by a concerned expert. Economic Journal 114(497), 487505.

Carroll, C. D., Overland, J.R., and Weil, D. N. (1997). Comparison utility in a growth model. Journal of Economic Growth 2, 33967.

Case, A., and Deaton, A.S. (2005). Broken down by work and sex: How our health declines. In: Analyses in the Economics of Aging, University of Chicago Press, 185-212.

CES (2017). Consumer Expenditure Survey. U.S. Bureau of Labor Statistics. https://www.bls.gov/cex/2016/CrossTabs/singlesbyinc/malesinc.PDF

Chetty, R. (2006). A new method of estimating risk aversion. American Economic Review 96, 1821-1834.

Cuypers, M., Lamers, R.E.D., Cornel, E.B., van de Poll-Franse, L.V., de Vries, M., and Kil, P.J.M. (2017). The impact of prostate cancer diagnosis and treatment decision-making on health-related quality of life before treatment onset. Support Care Cancer, University of Chicago Press, 185-212.

Dalgaard, C-J., and Strulik, H. (2014). Optimal aging and death: Understanding the Preston Curve. Journal of the European Economic Association 12, 672-701.

Dalgaard, C-J., and Strulik, H. (2017). The genesis of the golden age - accounting for the rise in health and leisure. Review of Economic Dynamics 24, 132-151.

Finkelstein, A., Luttmer, E.F., and Notowidigdo, M.J. (2013). What good is wealth without health? The effect of health on the marginal utility of consumption. Journal of the European Economic Association 11(1), 221-258. 
Grossman, M. (1972). On the concept of health capital and the demand for health. Journal of Political Economy 80, 223-255.

Honiden, S., Sundaram, V., Nease, R.F., Holodniy, M., Lazzeroni, L.C., Zolopa, A., and Owens, D.K. (2006). The effect of diagnosis with HIV infection on health-related quality of life. Quality of Life Research 15, 69-82.

Jorda, O., Knoll, K., Kuvshinov, D., Schularick, M., and Taylor, A.M. (2017). The Rate of Return on Everything, 18702015. National Bureau of Economic Research Working Paper w24112

Koszegi, B. (2003). Health anxiety and patient behavior. Journal of Health Economics 22, 1073-1084.

Koszegi, B. (2006). Emotional agency. Quarterly Journal of Economics 121(1), 121-155.

Layard, R., Mayraz, G., and Nickell, S. (2008). The marginal utility of income. Journal of Public Economics 92, 1846-1857.

Lazarus, R. (1966). Psychological Stress and the Coping Process. New York: McGraw Hill.

Lerman, C., Narod, S., Schulman, K., Hughes C., Gomez-Caminero A., Bonney G., Gold K., et al. (1996). BRCA1 Testing in Families with Hereditary Breast-Ovarian Cancer. A Prospective Study of Patient Decision Making and Outcomes. Journal of the American Medical Association 275(24), 1885-1892.

Loewenstein, G. (1987). Anticipation and the valuation of delayed consumption. Economic Journal 97, 666-684.

MEPS (2010), U.S. Department of Health \& Human Services. Center for Financing, Access and Cost Trends, Agency for Healthcare Research and Quality: Medical Expenditure Panel Survey, 2010 (https://meps . ahrq.gov/mepsweb/data_stats/quick_tables_results.$j$ sp? component= 1\&subcomponent=0\&year=-1\&tableSeries $=1 \&$ searchText=\&searchMethod=1\&Action=Search)

Mitnitski, A.B., Mogilner, A.J., MacKnight, C., and Rockwood, K. (2002). The accumulation of deficits with age and possible invariants of aging. Scientific World 2, 1816-1822.

Mitnitski, A.B., Mogilner, A.J., MacKnight, C., and Rockwood, K. (2002b). The mortality rate as a function of accumulated deficits in a frailty index. Mechanisms of ageing and development $123,1457-1460$.

Monteiro, G. and Turnovsky S.J. (2016). Anticipated consumption and its impact on capital accumulation and growth: "Forward-looking" versus "backward-looking" consumption reference. International Journal of Economic Theory 12, 203-232.

Moran, Molly J. and Monje, Carlos (2016). Guidance on Treatment of the Economic Value of Statistical Life (VSL) in U.S. Department of Transportation Analyses - 2016 Adjustment. U.S. Department of Transportation. 
Murphy, K.M., and Topel, R.H. (2006). The value of health and longevity. Journal of Political Economy 114, 871-904.

Nair, K.S. (2005). Aging muscle, American Journal of Clinical Nutrition 81, 953-963.

Nakagawa, F., May, M., and Phillips, A. (2013). Life expectancy living with HIV: recent estimates and future implications. Current Opinion in Infectious Diseases 26 (1), 17-25.

NVSS (2014). US department of health and human services, national vital statistics system. National Vital Statistics Report 63(7).

OECD (2018). Health spending (indicator). OECD (2018), Health spending (indicator). doi: 10.1787/8643de7e-en (Accessed on 26 March 2018).

Oster, E., Shoulson, I., and Dorsey, R. (2013a). Optimal expectations and limited medical testing: Evidence from Huntington Disease. American Economic Review 103(2), 804-830.

Oster, E., Shoulson, I., and Dorsey, R. (2013b). Limited life expectancy, human capital and health investments. American Economic Review 103(5), 19772002.

Roberts, J. Scott, Barber, M., Brown, T.M., Cupples, L.A., Farrer, L.A., LaRusse, S.A., Post, S.G., et al. (2004). Who seeks genetic susceptibility testing for Alzheimer's Disease? Findings from a multisite randomized clinical trial. Genetics in Medicine 6(4), 197-203.

Ropka, Mary, Jennifer Wenzel, Elayne Phillips, Mir Siadaty, and John Philbrick (2006). Uptake rates for breast cancer genetic testing: A systematic review. Cancer Epidemiology, Biomarkers and Prevention, 15(5) 840-844.

Rockwood, K., and Mitnitski, A. (2007). Frailty in relation to the accumulation of deficits. The Journals of Gerontology Series A: Biological Sciences and Medical Sciences 62(7), 722-727.

Schünemann, J., Strulik, H., Trimborn, T. (2017a). Going from bad to worse: Adaptation to poor health, health spending, longevity, and the value of life. Journal of Economic Behavior and Organization, 140, 130-146.

Schünemann, J., Strulik, H., Trimborn, T. (2017b). The gender gap in mortality: How much Is explained by behavior? Journal of Health Economics, 54, 79-90.

Schünemann, J., Strulik, H., Trimborn, T. (2017c). The marriage gap: Optimal aging and death in partnerships. Center for European, Governance and Economic Development Research Discussion Papers, 339.

Shoulson, I., and Young, A. (2011). Milestones in Huntington Disease. Movement Disorders 26(6), 1127-1133.

Skirbekk, V. (2004), Age and individual productivity: A literature survey, Vienna Yearbook of Population Research, 133-153.

Strulik, H. (2015). Frailty, mortality, and the demand for medical care, Journal of the Economics of Ageing 6, 5-12. 
Strulik, H. (2018a). The Return to education in terms of wealth and health. Journal of the Economics of Ageing 12, 1-14.

Thornton, R. L. (2008). The demand for, and impact of, learning HIV status. American Economic Review 98(5), 18291863. 